\title{
Approximation of Reachable Sets by Direct Solution Methods of Optimal Control Problems
}

\author{
Robert Baier, Christof Büskens ${ }^{\dagger}$ Ilyes Aïssa Chahma; Matthias Gerdts ${ }^{\S}$
}

13th December 2004

\begin{abstract}
A numerical method for the approximation of reachable sets of linear control systems is discussed. The method is based on the formulation of suitable optimal control problems with varying objective function, whose discretization by Runge-Kutta methods lead to finite dimensional convex optimization problems. It turns out that the order of approximation for the reachable set depends on the particular choice of the Runge-Kutta method in combination with the selection strategy used for control approximation. For an inappropriate combination the expected order of convergence can not be achieved in general. The method is illustrated by two examples using different Runge-Kutta methods and selection strategies and allows to estimate the order of convergence numerically.
\end{abstract}

Keywords: optimal control, approximation of reachable sets, direct solution methods, order of convergence

${ }^{*}$ Department of Mathematics, University of Bayreuth, 95440 Bayreuth, Germany, Robert.Baier@uni-bayreuth.de

${ }^{\dagger}$ Department of Mathematics, University of Bremen, 28344 Bremen, Germany

$\ddagger$ Cetelem Bank GmbH, Schwanthalerstrasse 31, 80336 München, Germany, a.chahma@cetelembank.de

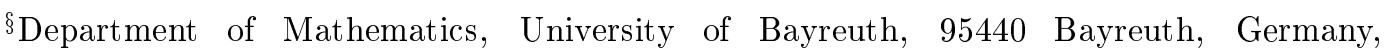
Matthias.Gerdts@uni-bayreuth.de 


\section{Introduction}

The subject of this paper is the description of an algorithm for the approximation of reachable sets of linear control problems. The problem of determining convex reachable sets can be equivalently described by infinitely many optimal control problems, where the objective function is adapted. By choosing only finitely many directions approximations of reachable sets can be obtained. The occuring optimal control problems are not solved theoretically by use of the Pontryagin's maximum principle as in [38] but numerically by suitable discretization methods. This allows to treat also time dependent linear problems and even nonlinear ones. Non-polyhedral control regions can be treated as nonlinear inequalities and equalities. Results concerning the convergence of discretized optimal control problems can be found in [30], [10] and the references stated therein.

In this context, the particular choice of the selection strategy used for control approximation turns out to be crucial for the order of convergence and depends on the choice of the Runge-Kutta scheme used for the discretization of the underlying differential equations. In order to illustrate this dependency several Runge-Kutta methods with different selection strategies (piecewise constant, piecewise linear, independent selection) are discussed in more detail for two illustrative examples.

By this approach cumbersome set operations (like Minkowski sums, unions of sets, ...) can be avoided and lead to known optimization methods, which in addition yield not only the endpoints of optimal trajectories, but the entire trajectory including the corresponding optimal control. Furthermore, this approach is useful for linear control problems with control regions formulated with nonlinear restrictions (see (7)) and in nonlinear control problems yielding convex reachable sets, too. However, the close connection between set-valued analysis and optimal control is shown in Section 3. A comparison with set-valued methods as in $[12,4,3,41,8]$ is beyond the scope of this paper.

Methods for linear differential inclusions based on set-valued quadrature methods or set-valued Runge-Kutta methods are mentioned in [3] as well as other methods, e.g. estimation methods for reachable sets (cf. [15]) and ellipsoidal methods (cf. [23] for an overview). Newer developments of these methods achieve inner approximations ([24], [26]) and outer approximations [25] of the reachable set (see also [4]).

The problem of the approximation of reachable sets appears in several disciplines: control theory, ordinary differential equations with uncertainties or with discontinuities in the state, necessary conditions for a minimum in nonsmooth analysis, differential games and viability theory, cf. [5], [1], [33], [14]. The convexity of these reachable sets can be guaranteed for linear differential inclusions, but may also appear for nonlinear problems.

The paper is organized as follows. In Section 2 basic notations and properties of reachable sets are summarized. Basic facts on the description of convex sets and arithmetic set operations are introduced and form the basis for the re- 
sults of Section 3. In particular, the Hausdorff and the Demyanov distances are defined, which are used to measure the speed of convergence w.r.t. the optimal value and the optimal trajectory, respectively. In Section 3 the problem of calculating the boundary of the reachable set is reformulated as infinitely many optimal control problems which differ only in the objective function. These optimal control problems are discretized by use of explicit Runge-Kutta methods and suitable control approximations resulting in finite dimensional (linear/nonlinear) optimization problems. Herein, several approximation classes for the control lead to different selection strategies in the discretization. The section ends with a formulation of the proposed method for the approximation of reachable sets and its implementation. Several combinations of Runge-Kutta methods and selection strategies are discussed in Section 4 with illustrative examples. Tables with convergence results and visualizations of reachable sets are included. Finally, an outline for further research concludes the paper.

\section{Notation}

In this section, some introductory definitions and results are collected.

The basic underlying problem is the following control problem:

Problem 2.1 Let $A(\cdot): \mathbb{R}^{n} \rightarrow \mathbb{R}^{n \times n}$ and $B(\cdot): \mathbb{R}^{m} \rightarrow \mathbb{R}^{m \times n}$ be two L $L_{1}$-integrable matrix functions.

Let $U \subset \mathbb{R}^{m}$ be a nonempty, convex compact set and $I:=\left[t_{0}, t_{f}\right]$ be a real interval. For a given control function $u: I \rightarrow \mathbb{R}^{m}$ with $u(\cdot) \in L_{\infty}\left(I, \mathbb{R}^{m}\right)$ we are looking for a solution $x(\cdot) \in W^{1, \infty}\left(I, \mathbb{R}^{n}\right)$ of the differential equation

$$
\begin{aligned}
\dot{x}(t) & =A(t) x(t)+B(t) u(t) & & \text { (a.e. } t \in I), \\
x\left(t_{0}\right) & =x_{0}, & & \\
u(t) & \in U & & \text { (a.e. } t \in I) .
\end{aligned}
$$

Definition 2.2 Let us study Problem 2.1 and let $t \in I$. Then,

$$
\begin{array}{r}
\mathcal{R}\left(t, t_{0}, x_{0}\right):=\left\{y \in \mathbb{R}^{n} \mid \exists u(\cdot) \text { control function and } \exists x(\cdot)\right. \text { corresponding } \\
\text { solution of Problem 2.1 with } x(t)=y\}
\end{array}
$$

is called the reachable set of the corresponding control problem for the time $t$.

In 1965, Aumann discovered the convexity of the set-valued integral in [2] which easily leads to the convexity of the reachable set for linear control problems.

Proposition 2.3 In Problem 2.1, the reachable set $\mathcal{R}\left(t, t_{0}, x_{0}\right)$ is convex, compact and nonempty for every $t \in I$. 
Proof: see e.g. [37, Theorem 1]

Some notations from Convex Analysis are recalled which are necessary for the explanation of the algorithm described later.

Definition 2.4 Denote by $\mathcal{C}\left(\mathbb{R}^{n}\right)$ the set of all nonempty convex compact sets in $\mathbb{R}^{n}$ and let $C \in \mathcal{C}\left(\mathbb{R}^{n}\right)$ and $l \in \mathbb{R}^{n}$.

Then,

$$
\delta^{*}(l, C):=\max _{c \in C} l^{\top} c
$$

is the support function of $C$ in direction $l$ and

$$
Y(l, C):=\left\{c \in C \mid l^{\top} c=\delta^{*}(l, C)\right\}
$$

is the set of supporting points of $C$ in direction $l$.

We need the following property of support functions:

Lemma 2.5 Let $C=C_{1} \times C_{2} \in \mathcal{C}\left(\mathbb{R}^{n}\right)$ with convex sets $C_{i} \subset \mathbb{R}^{n_{i}}, n_{i} \in$ $\{1, \ldots, n\}, i=1,2$, and $n_{1}+n_{2}=n$. Then, for given $l=\left(l_{1}^{\top}, l_{2}^{\top}\right)^{\top} \in \mathbb{R}^{n}$ with $l_{i} \in \mathbb{R}^{n_{i}}$, $i=1,2$, we have:

$$
\delta^{*}(l, C)=\delta^{*}\left(l_{1}, C_{1}\right)+\delta^{*}\left(l_{2}, C_{2}\right) .
$$

Proof: see e.g. [19, §V, Discussion after Remark 3.3.6]

Support functions resp. supporting points describe fully a convex compact set.

Proposition 2.6 Let $C \in \mathcal{C}\left(\mathbb{R}^{n}\right)$. Then,

$$
\begin{aligned}
& C=\bigcap_{\|l\|_{2}=1}\left\{x \in \mathbb{R}^{n} \mid l^{\top} x \leq \delta^{*}(l, C)\right\}, \quad \partial C=\bigcup_{\|l\|_{2}=1} Y(l, C), \\
& C=\operatorname{co}\left(\bigcup_{\|l\|_{2}=1}\{y(l, C)\}\right) \text { with arbitrary } y(l, C) \in Y(l, C),
\end{aligned}
$$

where $\partial C$ denotes the boundary of $C$ and $\operatorname{co}(\cdot)$ denotes the convex hull of a set.

Proof: see e.g. [19, §V., Theorem 2.2.2] and [19, §V., Proposition 3.1.5]. The last equation follows easily, if one estimates the support function of the right-hand side in direction $\eta$ by $\eta^{\top} y(\eta, C)=\delta^{*}(\eta, C)$ from below.

A common arithmetic operations on sets is the scalar multiplication and the Minkowski sum which are recalled here. 
Definition 2.7 Let $C, D \in \mathcal{C}\left(\mathbb{R}^{n}\right), \lambda \in \mathbb{R}$ and $A \in \mathbb{R}^{m \times n}$. Then,

$$
\lambda C:=\{\lambda c \mid c \in C\}
$$

defines the scalar multiplication,

$$
A C:=\{A c \mid c \in C\}
$$

the image of $C$ under the linear map $x \mapsto A x$ and

$$
C+D:=\{c+d \mid c \in C, d \in D\}
$$

the Minkowski sum.

We need the following theoretical result which states convexity and compactness of the set operations defined above.

Lemma 2.8 Let $C, D \in \mathcal{C}\left(\mathbb{R}^{n}\right), \lambda \in \mathbb{R}$ and $A \in \mathbb{R}^{m \times n}$. Then, $\lambda C$ and $C+D$ are elements of $\mathcal{C}\left(\mathbb{R}^{n}\right)$ and $A C$ is an element of $\mathcal{C}\left(\mathbb{R}^{m}\right)$. Furthermore,

$$
\begin{aligned}
\delta^{*}(l, \lambda C) & =\lambda \delta^{*}(l, C), & Y(l, \lambda C) & =\lambda Y(l, C) \\
\delta^{*}(\eta, A C) & =\delta^{*}\left(A^{\top} \eta, C\right), & Y(\eta, A C) & =A Y\left(A^{\top} \eta, C\right), \\
\delta^{*}(l, C+D) & =\delta^{*}(l, C)+\delta^{*}(l, D), & Y(l, C+D) & =Y(l, C)+Y(l, D)
\end{aligned}
$$

for all $l \in \mathbb{R}^{n}, \eta \in \mathbb{R}^{m}$.

Proof: To guarantee that the operations give results in $\mathcal{C}\left(\mathbb{R}^{n}\right)$ and the equations on the support functions see [19, $\S \mathrm{V}$, Theorem 3.3.3(i) and Proposition 3.3.4]. The equations on the supporting set follow immediately from calculus rules on the subdifferential in [19, §VI, Theorem 4.1.1 and equation (3.1)] and [32, Theorem 23.9], since [19, §VI, Proposition 2.1.5 and equation (3.1)] connects the subdifferential of the support function and the supporting set.

Definition 2.9 Let $C, D \in \mathcal{C}\left(\mathbb{R}^{n}\right)$. Then,

$$
\begin{aligned}
\mathrm{d}(C, D) & :=\max _{c \in C} \min _{d \in D}\|c-d\|_{2}, \\
\mathrm{~d}_{H}(C, D) & :=\max \{\mathrm{d}(C, D), \mathrm{d}(D, C)\}
\end{aligned}
$$

are defining the one-sided Hausdorff distance resp. the Hausdorff distance of the two sets.

The Demyanov distance between two sets is defined as

$$
\mathrm{d}_{D}(C, D):=\sup _{l \in T_{C} \cap T_{D}}\|y(l, C)-y(l, D)\|_{2},
$$

where $T_{C}$ is defined as set of all normed directions in $\mathbb{R}^{n}$ for which the supporting set $Y(l, C)$ consists of only one point $y(l, C)\left(T_{D}\right.$ is defined analogously for the set $D) . T_{C}$ and $T_{D}$ are subsets of the unit sphere of full measure. 
Well-known properties of the support function make it easy to prove the following result for the Hausdorff-distance:

Proposition 2.10 Let $C, D \in \mathcal{C}\left(\mathbb{R}^{n}\right)$. Then,

$$
\mathrm{d}_{H}(C, D)=\max _{|l| \|_{2}=1}\left|\delta^{*}(l, C)-\delta^{*}(l, D)\right| \leq \mathrm{d}_{D}(C, D) .
$$

Proof: see e.g. [19, §V, Theorem 3.3.8] and [9, Lemma 4.1]

\section{New Method for the Approximation of Reach- able Sets}

\subsection{Computation of the Reachable Set by Optimal Con- trol}

Since we know from Proposition 2.3 that the reachable set for problem 2.1 is convex, it is sufficient to calculate merely the boundary of the reachable set.

Proposition 2.6 gives a motivation to calculate at least one support point (which lies automatically at the boundary) of the reachable set in direction $l \in \mathbb{R}^{n}$ with $\|l\|_{2}=1$. Note that even in the case that the reachable set is not strictly convex and the set of supporting points is a $(n-1)$-dimensional face, for a fixed direction $l$, one supporting point in this direction is sufficient to reconstruct the reachable set.

Thus, to calculate a supporting point $x\left(t_{f}\right)$ on the boundary of the reachable set $\mathcal{R}\left(t_{f}, t_{0}, x_{0}\right)$ in a fixed direction $l$ we have to find an admissible control function $u(t) \in U$ that maximizes the functional $y \mapsto l^{\top} y$ (resulting in the support function $\delta^{*}\left(l, \mathcal{R}\left(t_{f}, t_{0}, x_{0}\right)\right)$ as optimal value). This constitutes the following special optimal control problem of Mayer type:

$$
\left(\mathbf{O C P}_{\mathbf{1}}\right) \quad \begin{cases}\text { Maximize } & l^{\top} x\left(t_{f}\right) \\ \text { w.r.t. } & u \in L^{\infty}\left(\left[t_{0}, t_{f}\right], \mathbb{R}^{m}\right), x \in W^{1, \infty}\left(\left[t_{0}, t_{f}\right], \mathbb{R}^{n}\right) \\ & x(\cdot) \text { corresponding solution to } u(\cdot) \text { for }(1 \mathrm{a})-(1 \mathrm{c}) .\end{cases}
$$

We denote the optimal solution of $\left(O C P_{l}\right)$ by $x^{\star}(t ; l)$ and $u^{\star}(t ; l)$, where the argument $l$ indicates the dependency of the direction $l$.

As already mentioned in Proposition 2.6, the convexity and compactness of the reachable set guaranteed by Proposition 2.3 leads to the equivalent representation by considering supporting points in all directions $l \in \mathbb{R}^{n},\|l\|_{2}=1$ :

$$
\mathcal{R}\left(t_{f}, t_{0}, x_{0}\right)=\operatorname{co}\left\{x^{\star}\left(t_{f} ; l\right) \mid l \in \mathbb{R}^{n},\|l\|_{2}=1\right\} .
$$




\subsection{Approximation of Reachable Sets by Discretized Op- timal Control Problems}

In general, for complex problems neither we can compute a solution of $\left(O C P_{l}\right)$ analytically nor for all directions $l$. Hence, we suggest to approximate $\left(O C P_{l}\right)$ numerically and consider only a finite number of directions $l_{i}, i=1, \ldots, M:=N_{l}$. This yields an approximation

$$
\mathcal{R}_{M}\left(t_{f}, t_{0}, x_{0}\right) \approx \mathcal{R}\left(t_{f}, t_{0}, x_{0}\right)
$$

of the reachable set which will be specified hereafter.

For the moment let $l$ be fixed with $\|l\|_{2}=1$.

For $N_{t} \in \mathbb{N}, N_{t} \geq 2$ we introduce a grid with grid points

$$
t_{i}=t_{0}+i h \in\left[t_{0}, t_{f}\right], i=0,1, \ldots, N:=N_{t}, h=\frac{t_{f}-t_{0}}{N_{t}} .
$$

The control function $u(t)$ is discretized on each subinterval $\left[t_{i}, t_{i+1}\right]$ by the approximation

$$
u_{\text {app }}^{(i)}(t ; \hat{\mathbf{u}}), \quad t \in\left[t_{i}, t_{i+1}\right],
$$

where $\hat{\mathbf{u}}=\left(u_{0}, u_{1}, \ldots, u_{P-1}\right)^{\top} \in U^{P}$ is a finite dimensional vector parametrizing the selection strategy for the control in the following explicit Runge-Kutta scheme.

Let us first define explicit Runge-Kutta schemes before we will discuss particular strategies for the approximation of the control in more details. Each explicit Runge-Kutta scheme can be characterized by its Butcher array:

$$
\begin{array}{c|cccc}
\gamma_{1} & 0 & \cdots & \cdots & 0 \\
\gamma_{2} & \alpha_{21} & 0 & \cdots & 0 \\
\vdots & \vdots & \ddots & \ddots & \vdots \\
\gamma_{s} & \alpha_{s 1} & \cdots & \alpha_{s, s-1} & 0 \\
\hline & \beta_{1} & \cdots & \beta_{s-1} & \beta_{s}
\end{array}
$$

For a given control approximation $u_{a p p}^{(i)}(t ; \hat{\mathbf{u}})$ on $\left[t_{i}, t_{i+1}\right]$ a state approximation $x_{a p p}(t ; \hat{\mathbf{u}})$ is obtained via an explicit $s$-step Runge-Kutta discretization scheme:

$$
\begin{aligned}
x_{a p p}\left(t_{i+1} ; \hat{\mathbf{u}}\right) & =x_{a p p}\left(t_{i} ; \hat{\mathbf{u}}\right)+h \Phi\left(x_{a p p}\left(t_{i} ; \hat{\mathbf{u}}\right), \hat{\mathbf{u}}, h\right), \quad i=0,1, \ldots, N_{t}-1 \\
x_{a p p}\left(t_{0} ; \hat{\mathbf{u}}\right) & =x_{0}
\end{aligned}
$$

and

$$
\begin{aligned}
& \Phi\left(x_{a p p}\left(t_{i} ; \hat{\mathbf{u}}\right), \hat{\mathbf{u}}, h\right):=\sum_{j=1}^{s} \beta_{j}\left(A\left(t_{i}+\gamma_{j} h\right) \eta_{i+1}^{(j)}+B\left(t_{i}+\gamma_{j} h\right) u_{a p p}^{(i)}\left(t_{i}+\gamma_{j} h ; \hat{\mathbf{u}}\right)\right), \\
& \eta_{i+1}^{(j)}:=x_{a p p}\left(t_{i} ; \hat{\mathbf{u}}\right)+h \sum_{k=1}^{j-1} \alpha_{j k}\left(A\left(t_{i}+\gamma_{k} h\right) \eta_{i+1}^{(k)}+B\left(t_{i}+\gamma_{k} h\right) u_{a p p}^{(i)}\left(t_{i}+\gamma_{k} h ; \hat{\mathbf{u}}\right)\right) .
\end{aligned}
$$


Suitable values for the coefficients $\alpha_{j k}, \beta_{j}$ and $\gamma_{j}, 1 \leq j, k \leq s$ can be found in [7].

Let us now consider examples for selection strategies used in Section 4.

(i) Continuous and piecewise linear approximation:

$$
u_{a p p}^{(i)}(t ; \hat{\mathbf{u}}):=u_{i}+\frac{t-t_{i}}{h}\left(u_{i+1}-u_{i}\right) \quad \text { for } t \in\left[t_{i}, t_{i+1}\right], i=0,1, \ldots, N-1,
$$

with $P=N+1$.

(ii) Piecewise constant approximation:

$$
u_{a p p}^{(i)}(t ; \hat{\mathbf{u}}):=u_{i} \quad \text { for } t \in\left[t_{i}, t_{i+1}\right], i=0,1, \ldots, N-1,
$$

with $P=N$.

(iii) Independent selections at intermediate grid points $t_{i}+\gamma_{j} h$ :

$$
u_{a p p}^{(i)}\left(t_{i}+\gamma_{j} h ; \hat{\mathbf{u}}\right):=u_{i \cdot s+j-1}, \quad i=0,1, \ldots, N-1, j=1, \ldots, s,
$$

with $P=s \cdot N$. Here, each grid point creates a new independent selection for each subinterval. For modified Euler's method (see Section 4 and Figure 4 in Example 4.2) $\gamma_{1}=0, \gamma_{2}=\frac{1}{2}$ so that two independent selections $u_{2 i}$ and $u_{2 i+1}$ are chosen from $U$ for this method in each subinterval $\left[t_{i}, t_{i+1}\right]$. For Heun's method (see Section 4 and Figure 3 in Example 4.2) $\gamma_{1}=0$, $\gamma_{2}=1$ so that two independent selections $u_{2 i}$ and $u_{2 i+1}$ are also chosen from $U$ for this method in each subinterval $\left[t_{i}, t_{i+1}\right]$, although $t_{i}+\gamma_{2} h=t_{i+1}+\gamma_{1} h$ for $i=0, \ldots, N-1$.

Please notice, that further selection strategies are possible, e.g. independent selections with additional continuity constraints at the inner grid points $t_{i}, i=$ $1, \ldots, N-1$, or additional equality constraints at those intermediate grid points $t_{i}+\gamma_{j} h$ where different indices $j$ produce the same intermediate grid point (i.e., points where $\gamma_{j}=\gamma_{k}$ with $j \neq k$ ).

Thus, by this discretization the infinite dimensional optimal control problem $\left(O C P_{l}\right)$ is approximated by the finite dimensional convex programming problem

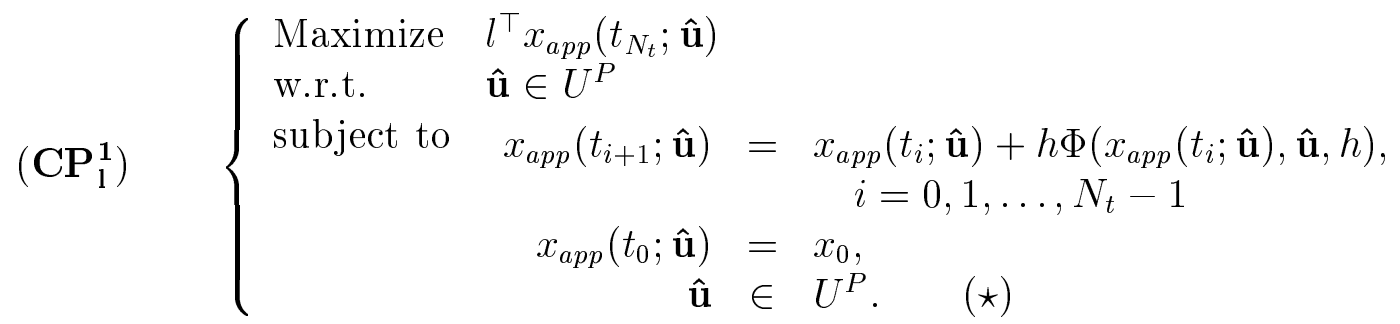

Notice, that $\hat{\mathbf{u}}$ implicitly defines a control approximation $u_{a p p}^{(i)}(\cdot ; \hat{\mathbf{u}})$ on each subinterval $\left[t_{i}, t_{i+1}\right]$, compare the examples (i)-(iii). 
We denote the optimal solution of $\left(C P_{l}^{1}\right)$ by $\hat{\mathbf{u}}^{\star}$.

If the conditions $(\star)$ can be written with a finite number of affine inequalities, $\left(C P_{l}^{1}\right)$ is a linear programming problem and called $\left(L P_{l}^{1}\right)$, otherwise a nonlinear (convex) programming problem.

In the sequel, we investigate the simplest case, the Euler's method In the sequel, we investigate the simplest case, the Euler's method with piecewise constant control approximation, since it is then easier possible to derive explicit solutions for the finite dimensional problems $\left(C P_{l}^{1}\right)$. Nevertheless, every explicit RungeKutta methods with the selection strategies (i)-(iii) will give a similar (more complicated) representation. The explicit formulae for the solution stress the strong connection to set-valued methods e.g. in [12, 4, 41] via support functions resp. supporting points.

In the case of Euler, (3) reduces to

$$
\Phi\left(x_{a p p}\left(t_{i} ; \hat{\mathbf{u}}\right), \hat{\mathbf{u}}, h\right)=A\left(t_{i}\right) x_{a p p}\left(t_{i} ; \hat{\mathbf{u}}\right)+B\left(t_{i}\right) u_{i} .
$$

The recursive evaluation in (3) for Euler's method yields

$$
x_{a p p}\left(t_{N_{t}} ; \hat{\mathbf{u}}\right)=\left(\prod_{i=0}^{N_{t}-1} Q_{i}\right) x_{0}+h \sum_{k=0}^{N_{t}-1}\left(\prod_{i=k+1}^{N_{t}-1} Q_{i}\right) B_{k} u_{k}
$$

with $Q_{i}:=I+h A\left(t_{i}\right), B_{k}:=B\left(t_{k}\right)$ and the $n \times n$-identity matrix $I$. The matrix product $\prod$ is defined as

$$
\prod_{i=k}^{j} Q_{i}:=Q_{j} \cdot Q_{j-1} \cdots Q_{k}
$$

Introducing this expression for $x_{a p p}\left(t_{f} ; \hat{\mathbf{u}}\right)$ in $\left(L P_{l}^{1}\right)$ yields the linear program

$$
\left(\mathbf{L P}_{\mathbf{l}}^{\mathbf{2}}\right) \quad \begin{cases}\text { Maximize } & l^{\top}\left(\sum_{k=0}^{N_{t}-1}\left(\prod_{i=k+1}^{N_{t}-1} Q_{i}\right) B_{k} u_{k}\right) \\ \text { subject to } & u_{k} \in U, \quad k=0,1, \ldots, N_{t}-1 .\end{cases}
$$

Note that this linear program has the same solution $\hat{\mathbf{u}}$ as $\left(L P_{l}^{1}\right)$, whereas the optimal objective function values are different, since we neglected constant terms.

To compute the objective function in $\left(L P_{l}^{2}\right)$ very efficiently we introduce additional artificial variables

$$
\begin{aligned}
\lambda_{N_{t}}^{\top} & :=l^{\top}, \\
\lambda_{i}^{\top} & :=\lambda_{i+1}^{\top} Q_{i}=\lambda_{i+1}^{\top}+h \lambda_{i+1}^{\top} A_{i} .
\end{aligned}
$$

These artificial variables are calculated backward in time and correspond to the discretized adjoint variable of the optimal control problem $\left(O C P_{l}\right)$. 
Then, $\left(L P_{l}^{2}\right)$ can be replaced by

$$
\left(\mathbf{L P}_{\mathbf{1}}^{\mathbf{3}}\right) \quad \begin{cases}\text { Maximize } & \sum_{k=0}^{N_{t}-1} \lambda_{k+1}^{\top} B_{k} u_{k} \\ \text { subject to } & u_{k} \in U, \quad k=0,1, \ldots, N_{t}-1 .\end{cases}
$$

Lemma 2.8 gives us

$$
\sum_{k=0}^{N_{t}-1} \delta^{*}\left(\lambda_{k+1}, B_{k} U\right)=\sum_{k=0}^{N_{t}-1} \delta^{*}\left(B_{k}^{\top} \lambda_{k+1}, U\right)
$$

as optimal value of $\left(L P_{l}^{3}\right)$ and hence, $\left(u_{0}, u_{1}, \ldots, u_{N_{t}-1}\right)$ with the supporting points $u_{k} \in Y\left(B_{k}^{\top} \lambda_{k+1}, U\right)$ as one solution.

In the special of box constraints, that is $U=\left\{u \in \mathbb{R}^{m} \mid \underline{u} \leq u \leq \bar{u}\right\}$, we define $S_{k}^{\top}:=\left(S_{k}^{1}, \ldots, S_{k}^{m}\right):=\lambda_{k+1}^{\top} B_{k} \in \mathbb{R}^{m}$. Since the objective function

$$
\sum_{k=0}^{N_{t}-1} S_{k} u_{k}=\sum_{k=0}^{N_{t}-1} \sum_{j=1}^{m} S_{k}^{j} \cdot u_{k}^{j}
$$

is maximized, if each term $S_{k}^{j} \cdot u_{k}^{j}$ is maximized, the solution of $\left(L P_{l}^{3}\right)$ is given by

$$
u_{k}^{j}= \begin{cases}\underline{u}^{j}, & \text { if } S_{k}^{j}<0, \\ \bar{u}^{j}, & \text { if } S_{k}^{j}>0, \\ \text { arbitrary, } & \text { else. }\end{cases}
$$

for $j=1, \ldots, m, k=0, \ldots, N_{t}-1$.

\subsection{Discrete reachable sets}

Discrete reachable sets are the reachable sets of the discretized equations and could be defined as endpoints of discrete solutions of the following problem.

Given the data in Problem 2.1, the discretized problem depends on the choice of the set $\mathcal{U}_{\text {app }}$ of all discretized control functions and on the Runge-Kutta scheme.

Problem 3.1 For a time discretization (2) with step-size $h=\frac{t_{f}-t_{0}}{N_{t}}$ and a given discretized control function $u_{\text {app }}(\cdot, \hat{\mathbf{u}})$ we are looking for a solution $x_{a p p}(\cdot, \hat{\mathbf{u}})$ at the grid-points $t_{i}, i=0,1, \ldots, N_{t}$, with

$$
\begin{aligned}
& x_{a p p}\left(t_{i+1} ; \hat{\mathbf{u}}\right)= x_{a p p}\left(t_{i} ; \hat{\mathbf{u}}\right)+h \Phi\left(x_{a p p}\left(t_{i} ; \hat{\mathbf{u}}\right), \hat{\mathbf{u}}, h\right) \\
& \quad \text { for } i=0,1, \ldots, N_{t}-1, \\
& x_{a p p}\left(t_{0} ; \hat{\mathbf{u}}\right)= x_{0}, \\
& u_{i} \in U, \quad i=0,1, \ldots, N_{t} \\
& u_{a p p}(\cdot, \hat{\mathbf{u}}) \in \mathcal{U}_{a p p} .
\end{aligned}
$$


Definition 3.2 Consider Problem 3.1 with a time discretization (2) and let $i \in$ $\left\{0,1, \ldots, N_{t}\right\}$. Then,

$$
\begin{aligned}
\mathcal{R}_{N}\left(t_{i}, t_{0}, x_{0}\right):=\left\{y \in \mathbb{R}^{n}\right. & \mid \exists u_{\text {app }}(\cdot ; \hat{\mathbf{u}}) \text { discretized control function and } \\
& \exists x_{\text {app }}(\cdot ; \hat{\mathbf{u}}) \text { corresponding solution of Problem } 3.1 \\
& \text { with } \left.x_{a p p}\left(t_{i} ; \hat{\mathbf{u}}\right)=y\right\}
\end{aligned}
$$

is called the discrete reachable set of the corresponding discretized control problem for the time $t_{i}$.

The definition above shows that each optimizer of problem $\left(C P_{l}^{1}\right)$ (resp. the reformulation $\left.\left(L P_{l}^{3}\right)\right)$ is a supporting point of the discrete reachable set $\mathcal{R}_{N}\left(t_{f}, t_{0}, x_{0}\right)$ in direction $l$. The optimal value of problem $\left(C P_{l}^{1}\right)$ coincides with the support function $\delta^{*}\left(l, \mathcal{R}_{N}\left(t_{f}, t_{0}, x_{0}\right)\right)$. Proposition 2.6 shows that

$$
\begin{aligned}
& \mathcal{R}_{N}\left(t_{f}, t_{0}, x_{0}\right)=\bigcap_{\|l\|_{2}=1}\left\{x \in \mathbb{R}^{n} \mid l^{\top} x \leq l^{\top} x_{\mathrm{app}}\left(t_{f} ; \hat{\mathbf{u}}^{\star}\right)\right\}, \\
& \mathcal{R}_{N}\left(t_{f}, t_{0}, x_{0}\right)=\operatorname{co}\left(\bigcup_{\|l\|_{2}=1}\left\{x_{\mathrm{app}}\left(t_{f} ; \hat{\mathbf{u}}^{\star}\right)\right\}\right) .
\end{aligned}
$$

In practice, only a finite number of different normed directions $l^{i}, i=1, \ldots, M$, are chosen.

Proposition 3.3 Consider Problem 3.1 with a time discretization (2) and let $i \in$ $\left\{0,1, \ldots, N_{t}\right\}$. Then, the corresponding discrete reachable set is convex, compact and nonempty.

Proof: For a chosen discretized control function $u_{\text {app }}(\cdot, \hat{\mathbf{u}})$, the discrete solution is defined by (5). The discrete reachable set coincides with the union of all such discrete solutions for all feasible discretized control functions. In the case of Euler and linear approximation of the controls, this corresponds to the union over all vectors $\hat{\mathbf{u}} \in \mathbb{R}^{m(N+1)}$. Definition 2.7 shows that the discrete reachable set

$$
\mathcal{R}_{N}\left(t_{f}, t_{0}, x_{0}\right)=\left(\prod_{i=0}^{N_{t}-1} Q_{i}\right) x_{0}+h \sum_{k=0}^{N_{t}-1}\left(\left(\prod_{i=k+1}^{N_{t}-1} Q_{i}\right) B_{k}\right) U
$$

is a scaled Minkowski sum of linearly transformed convex sets $U$. Lemma 2.8 proves the wanted properties of the discrete reachable set.

\subsection{Implementation}

In the sequel, we briefly discuss some numerical methods, which are suitable for solving the discretized optimal control problem $\left(C P_{l}^{1}\right)$. Of course, the choice 
of an appropriate method depends on the explicit representation of the control region $U$. Hence, we restrict the discussion to convex control regions $U$ defined by

$$
U=\left\{u \in X \mid g_{i}(u) \leq 0, i=1, \ldots, r\right\},
$$

where $X:=\left\{u \in \mathbb{R}^{m} \mid \widetilde{A} u=b, u \geq 0\right\}$ with a matrix $\widetilde{A} \in \mathbb{R}^{p \times m}$ and the functions $g_{i}(\cdot), i=1, \ldots, r$, could be either linear or nonlinear.

Remark 3.4 In the case, that the support function or the supporting points of the convex control set $U$ are known, general control regions $U$ can be approximated in another way. Proposition 2.6 suggests to use the approximation

$$
U \approx \bigcap_{i=1, \ldots, M}\left\{x \in \mathbb{R}^{m} \mid \eta^{i^{\top}} x \leq \delta^{*}\left(\eta^{i}, U\right)\right\}
$$

resp.

$$
U \approx \operatorname{co}\left(\bigcup_{i=1, \ldots, M}\left\{y\left(\eta^{i}, U\right)\right\}\right) \text { with arbitrary } y\left(\eta^{i}, U\right) \in Y\left(\eta^{i}, U\right)
$$

Herein, the $M$ different normed directions $\eta^{i} \in \mathbb{R}^{m}$ should be chosen in an appropriate way in order to approximate the unit sphere. One method is to parametrize them by spherical coordinates and use equidistant partitions on the parameter intervals for the angles (see [3, Subsection 3.1.2]).

If the functions $g_{i}$ in (7) are affine linear, then problem $\left(C P_{l}^{1}\right)$ is a linear optimization problem and can be solved by the well-known simplex method or some interior point method, cf. [42], suitable for linear programs. In the special case of an Euler approximation and $U$ defined by box constraints only, a very efficient method is described in Section 3.2.

If the functions $g_{i}$ are convex and smooth, i.e. at least continuously differentiable, then the resulting problem $\left(C P_{l}^{1}\right)$ is a convex but nonlinear programming problem and the sequential quadratic programming (SQP) method is appropriate provided the functions $g_{i}$ are defined for infeasible points, cf. [34], [35], [18]. Alternatively, the method of feasible directions is applicable, especially, if the functions $g_{i}$ are only defined for admissible points, cf. [43].

If the functions $g_{i}$ are convex but nonsmooth, the bundle method respectively the bundle trust region method (BT-method) is suitable, cf. [28], [31], [21], [22], [36]. In addition, Kelly's cutting plane method is also applicable, cf. [20]. Notice, that the BT-method and the cutting plane method are closely related, cf. [21], [36]. 


\section{Examples}

In the sequel we refer to the optimal control problem $\left(O C P_{1}\right)$, the differential equation (1a)-(1b), the control constraint (1c), and the control approximations discussed in (i)-(iii) in Section 3.2.

The following Runge-Kutta methods are used for the numerical computation of reachable sets:

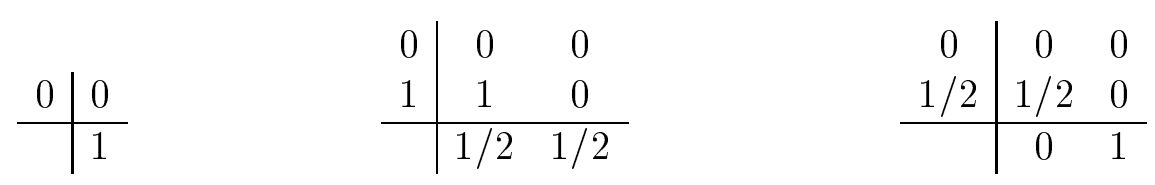

Euler's method Heun's method Modified Euler's method

For all numerical experiments the number of directions $M$ in Remark 3.4 is chosen as 1200???. For simplicity, the methods with different selection strategies are tested for time-independent two-dimensional problems (in which one could even calculate a theoretical solution for reference purposes). Nevertheless, the framework presented before is still valid and the methods could be used also in more complicated problems (time dependent and higher dimensional) met in practice.

From Definition 2.9 of the Hausdorff distance, it is clear that the approximation of the reachable set corresponds to a uniform convergence of the optimal value functions, whereas the approximation of trajectories corresponds to the uniform convergence of the maximizers and the Demyanov distance.

Example 4.1 (see [39, Example in section 4]) Let us consider the following example with $n=2, m=1, x_{0}=(0,0)^{\top}, I=[0,1], U=[0,1]$, and

$$
A(t)=\left(\begin{array}{ll}
0 & 1 \\
0 & 0
\end{array}\right), \quad B(t)=\left(\begin{array}{l}
0 \\
1
\end{array}\right) .
$$

In Figure 1 approximations to the reachable set $\mathcal{R}\left(1,0, x_{0}\right)$ are shown, in the left picture approximations with Euler's method with piecewise constant selections are shown (first order of convergence), in the right one the corresponding ones for Heun's method with continuous and piecewise linear control approximation (second order of convergence) are depicted. In both cases the set with the solid line shows the reference set (calculated with the corresponding method for $N=1280$ ). The dashed lines show the approximations for $N=10,20,40$ for Euler's method on the left picture (please note the halfening of the distance of the upper right corner of the sets when the number of subintervals is doubled). At the right one, the dashed lines show the approximations for $N=1,2,4$ for Heun's method ( $a$ smaller number of subintervals are chosen so that one could still see in Figure 1 a difference of the corresponding approximations). Please notice the more rapid 
convergence even for these small numbers of subintervals in comparision with Euler's method.
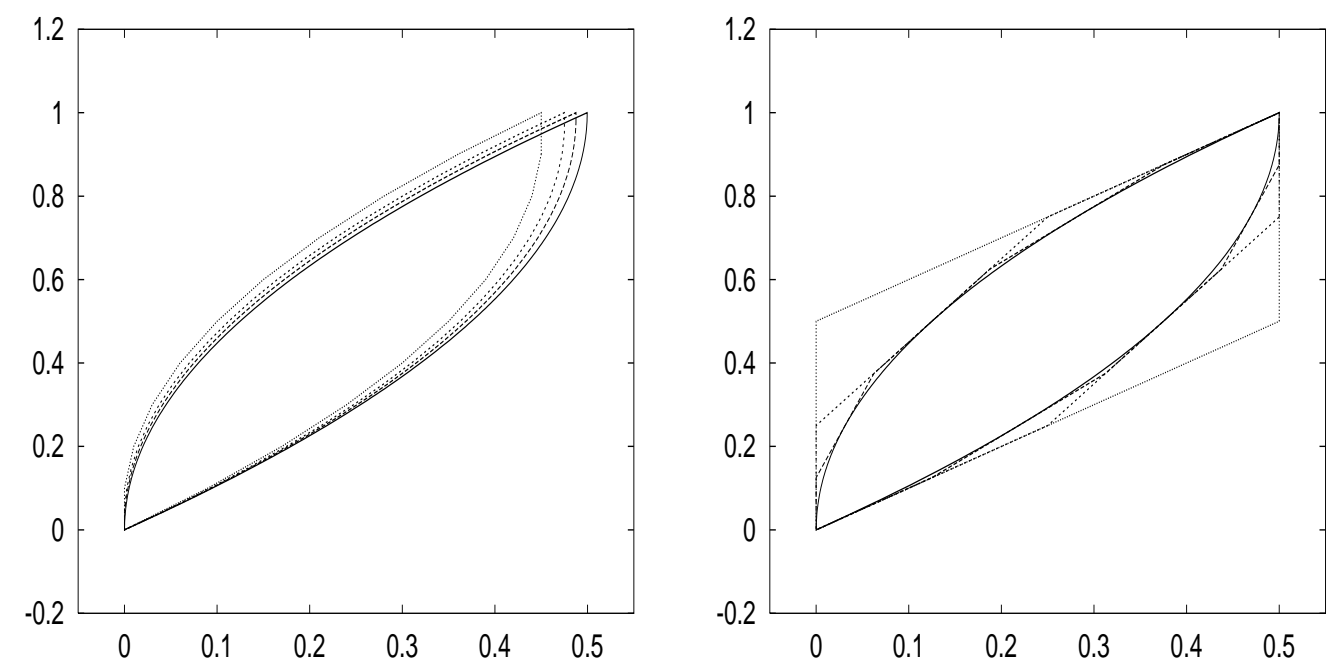

Figure 1: First order contra second order approximations to the reachable set (left: Euler's method with error $\mathcal{O}(h)$, right: Heun's method with error $\mathcal{O}\left(h^{2}\right)$ )

As Veliov explains in [39], the convergence of the trajectory could not be better than $\mathcal{O}(h)$ in this example. In Figure 2 the first order approximations to the control and to the state components (coordinates $x_{1}$ and $x_{2}$ ) are shown for Heun's method with continuous, piecewise linear selections. Again, the reference is computed by the method itself with $N=1280$ (solid line) and in dashed lines the approximations for $N=10,20,40$. As it is clearly seen, the order of convergence is only 1.
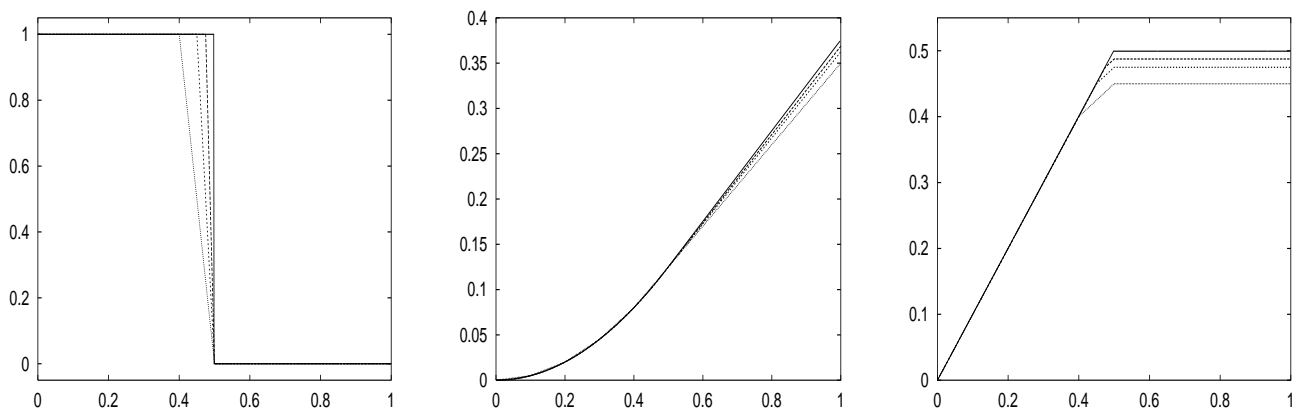

Figure 2: First order approximations to the control (left) and the state components (middle, right) by Heun's method

Here, the combination method of set-valued iterated trapezoidal rule together with Heun's method introduced in [3, 4] with $N=1000000$ serves as the reference 
set $\widehat{\mathcal{R}}_{\text {ref }}\left(0, x_{0},\right)$. By comparing the different values based on the optimal value function resp. the maximizers, the order of convergence is estimated. The angle $\varphi$ for the direction $l \in \mathbb{R}^{2}$, in which the maximum in (8) resp. (9) is attained, is shown in the most right column.

\begin{tabular}{r|c|c|r}
$N$ & $\begin{array}{c}\text { Hausdorff } \\
\text { distance }\end{array}$ & $\begin{array}{c}\text { estim. } \\
\text { order }\end{array}$ & angle \\
\hline 10 & 0.05000000 & NaN & 0.00500 \\
20 & 0.02500000 & 1.00000 & 0.00500 \\
40 & 0.01250000 & 1.00000 & 0.00500 \\
80 & 0.00625000 & 1.00000 & 0.00500 \\
160 & 0.00312500 & 1.00000 & 0.00500 \\
320 & 0.00156250 & 1.00000 & 0.00500 \\
640 & 0.00078125 & 1.00000 & 0.00500
\end{tabular}

\begin{tabular}{r|c|c|r}
$N$ & $\begin{array}{c}\text { Demyanov } \\
\text { distance }\end{array}$ & $\begin{array}{c}\text { estim. } \\
\text { order }\end{array}$ & angle \\
\hline 10 & 0.13702925 & NaN & 5.55500 \\
20 & 0.06806368 & 1.00953 & 5.55500 \\
40 & 0.03392323 & 1.00461 & 5.51500 \\
80 & 0.01731662 & 0.97012 & 5.53500 \\
160 & 0.00861479 & 1.00727 & 5.53500 \\
320 & 0.00426388 & 1.01465 & 5.53500 \\
640 & 0.00209303 & 1.02657 & 5.62500
\end{tabular}

Table I: order of convergence for Euler's method (left table: approximation of the reachable set, right table: approximation of the trajectories).

Table I shows the expected order of convergence 1 for reachable set and the trajectories. As remarked above the Hausdorff distance is attained at the upper right corner. This table shows the approximated values

$$
\max _{i=1, \ldots, M}\left|\delta^{*}\left(l_{i}, \mathcal{R}\left(1,0, x_{0}\right)\right)-\delta^{*}\left(l_{i}, \widehat{\mathcal{R}}_{r e f}\left(0, x_{0},\right)\right)\right|
$$

resp.

$$
\max _{i=1, \ldots, M}\left\|Y\left(l_{i}, \mathcal{R}\left(1,0, x_{0}\right)\right)-Y\left(l_{i}, \widehat{\mathcal{R}}_{r e f}\left(0, x_{0},\right)\right)\right\|_{2}
$$

at the chosen directions $l_{i}, i=1, \ldots, M$, for the two distances

$$
\mathrm{d}_{H}\left(\mathcal{R}\left(1,0, x_{0}\right), \mathcal{R}_{N}\left(1,0, x_{0}\right)\right) \quad \text { resp. } \quad \mathrm{d}_{D}\left(\mathcal{R}\left(1,0, x_{0}\right), \mathcal{R}_{N}\left(1,0, x_{0}\right)\right) .
$$

\begin{tabular}{r|c|c|r}
$N$ & $\begin{array}{c}\text { Hausdorff } \\
\text { distance }\end{array}$ & $\begin{array}{c}\text { estim. } \\
\text { order }\end{array}$ & angle \\
\hline 10 & 0.00124700 & NaN & 3.09500 \\
20 & 0.00031111 & 2.00295 & 3.12000 \\
40 & 0.00007788 & 1.99805 & 6.27500 \\
80 & 0.00001947 & 1.99990 & 3.14000 \\
160 & 0.00000488 & 1.99688 & 6.26000 \\
320 & 0.00000122 & 1.99929 & 3.14500 \\
640 & 0.00000030 & 2.00266 & 6.22500
\end{tabular}

\begin{tabular}{r|c|c|r}
$N$ & $\begin{array}{c}\text { Demyanov } \\
\text { distance }\end{array}$ & $\begin{array}{c}\text { estim. } \\
\text { order }\end{array}$ & angle \\
\hline 10 & 0.06636590 & NaN & 5.55500 \\
20 & 0.03273184 & 1.01975 & 5.55500 \\
40 & 0.01668369 & 0.97226 & 2.40000 \\
80 & 0.00848003 & 0.97630 & 5.53500 \\
160 & 0.00419649 & 1.01488 & 5.53500 \\
320 & 0.00205473 & 1.03024 & 5.53500 \\
640 & 0.00099208 & 1.05042 & 5.62500
\end{tabular}

Table II: order of convergence for Heun's method (left table: approximation of the reachable set, right table: approximation of the trajectories)

For Heun's method with continuous, piecewise linear control approximation, Table II shows order of convergence 2 for the reachable set and only order 1 for the trajectories. 
Example 4.2 (see [4, Example 4.4]) Let us consider the following example with $n=2, m=2, x_{0}=(0,0)^{\top}, I=[0,2], U=\left\{x \in \mathbb{R}^{2} \mid\|x\|_{2} \leq 1\right\}$, and

$$
A(t)=\left(\begin{array}{cc}
0 & 1 \\
-2 & -3
\end{array}\right), \quad B(t)=\left(\begin{array}{ll}
1 & 0 \\
0 & 1
\end{array}\right)
$$

This example introduces the nonlinear constraint

$$
u_{1}^{2}+u_{2}^{2} \leq 1
$$

for the control variable $u=\left(u_{1}, u_{2}\right)^{\top}$.

The second order approximations to the reachable set $\mathcal{R}\left(2,0, x_{0}\right)$ calculated by Heun's method with piecewise constant controls resp. with independent control selection in $t_{i}$ and $t_{i+1}$ (see (4)) are shown in Figure 3.
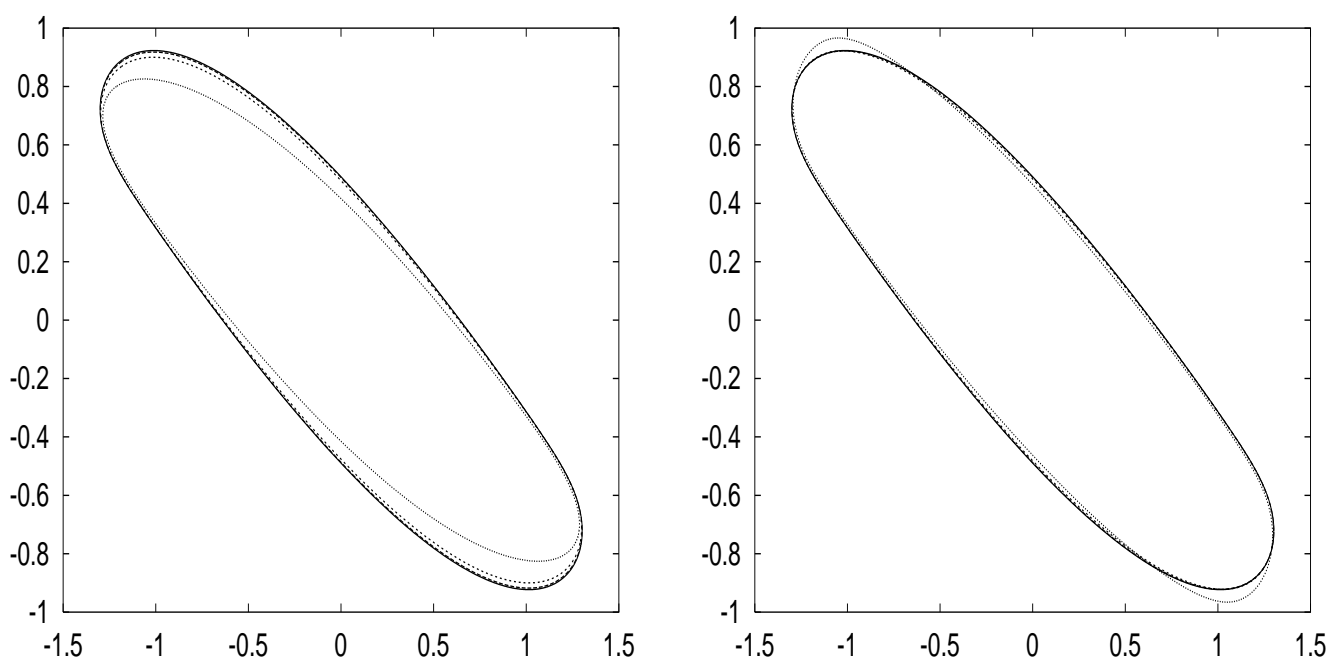

Figure 3: second order approximations to the reachable set for Heun's method with piecewise constant control approximation (left) resp. independent control selection (right).

The set with the solid line shows the reference set (calculated with the corresponding method for $N=160$ ) and the dashed lines represent the approximations for $N=5,10,20$. At the left picture the convergence order $\mathcal{O}\left(h^{2}\right)$ can be seen by studying the boundary of the sets near by $y=1$.

Both selection strategies seems to converge with order 2 which is assured by Tables III and IV.

Nevertheless, Figure 4 shows that the choice of the selection strategies for the control should depend on the Runge-Kutta method. In Figure 4 the piecewise constant selection strategy is compared with the independent control selections in $t_{i}$ and $t_{i}+\frac{h}{2}$ for modified Euler's method (see (4)). The latter selection strategy 


\begin{tabular}{r|r|r|c}
$N$ & $\begin{array}{r}\text { Hausdorff } \\
\text { distance }\end{array}$ & Order & angle \\
\hline 5 & 0.10328935 & $\mathrm{NaN}$ & 1.37000 \\
10 & 0.02307167 & 2.16250 & 1.53000 \\
20 & 0.00521186 & 2.14625 & 1.57500 \\
40 & 0.00123195 & 2.08086 & 4.73500 \\
80 & 0.00029922 & 2.04164 & 1.60000 \\
160 & 0.00007372 & 2.02105 & 4.74500
\end{tabular}

\begin{tabular}{r|r|r|c}
$N$ & $\begin{array}{r}\text { Demyanov } \\
\text { distance }\end{array}$ & $\begin{array}{r}\text { Order } \\
\text { trajectory }\end{array}$ & angle \\
\hline 5 & 0.37223126 & NaN & 0.90500 \\
10 & 0.07159599 & 2.37825 & 0.88500 \\
20 & 0.01535558 & 2.22112 & 4.02500 \\
40 & 0.00355544 & 2.11066 & 4.02500 \\
80 & 0.00085565 & 2.05493 & 4.02500 \\
160 & 0.00020992 & 2.02719 & 4.02500
\end{tabular}

Table III: Order of Convergence for Heun's method with piecewise constant control approximation.

\begin{tabular}{r|r|r|c}
$N$ & $\begin{array}{r}\text { Hausdorff } \\
\text { distance }\end{array}$ & Order & angle \\
\hline 5 & 0.04517018 & NaN & 1.72000 \\
10 & 0.00772443 & 2.54787 & 4.23500 \\
20 & 0.00203009 & 1.92789 & 4.30000 \\
40 & 0.00051385 & 1.98211 & 4.33500 \\
80 & 0.00012897 & 1.99429 & 1.21000 \\
160 & 0.00003229 & 1.99784 & 1.22000
\end{tabular}

\begin{tabular}{r|r|r|c}
$N$ & $\begin{array}{r}\text { Demyanov } \\
\text { distance }\end{array}$ & $\begin{array}{r}\text { Order } \\
\text { trajectory }\end{array}$ & angle \\
\hline 5 & 0.16781544 & NaN & 1.18500 \\
10 & 0.04611042 & 1.86371 & 0.87500 \\
20 & 0.01077148 & 2.09788 & 4.01500 \\
40 & 0.00257389 & 2.06520 & 0.87500 \\
80 & 0.00062808 & 2.03492 & 0.87500 \\
160 & 0.00015506 & 2.01802 & 4.01500
\end{tabular}

Table IV: Order of Convergence for Heun's method with independent selection strategy (iii).

destroys order of convergence 2 of the Runge-Kutta method. This is verified in the Tables $V$ (order $\mathcal{O}\left(h^{2}\right)$ ) and VI (only order $\mathcal{O}(h)$ ) for the convergence to the reachable set and the trajectories.

\begin{tabular}{r|r|r|c}
$N$ & $\begin{array}{r}\text { Hausdorff } \\
\text { distance }\end{array}$ & Order & angle \\
\hline 5 & 0.10328935 & NaN & 1.37000 \\
10 & 0.02307167 & 2.16250 & 1.53000 \\
20 & 0.00521186 & 2.14625 & 1.57500 \\
40 & 0.00123195 & 2.08086 & 4.73500 \\
80 & 0.00029922 & 2.04164 & 1.60000
\end{tabular}

\begin{tabular}{r|r|r|c}
$N$ & $\begin{array}{r}\text { Demyanov } \\
\text { distance }\end{array}$ & $\begin{array}{r}\text { Order } \\
\text { trajectory }\end{array}$ & angle \\
\hline 5 & 0.37223121 & NaN & 0.90500 \\
10 & 0.07159599 & 2.37825 & 0.88500 \\
20 & 0.01535559 & 2.22112 & 4.02500 \\
40 & 0.00355571 & 2.11056 & 4.02500 \\
80 & 0.00085566 & 2.05503 & 0.88500
\end{tabular}

Table V: Order of Convergence for the modified Euler's method with piecewise constant control approximation. 


\begin{tabular}{r|r|r|c}
$N$ & $\begin{array}{r}\text { Hausdorff } \\
\text { distance }\end{array}$ & Order & angle \\
\hline 5 & 0.83583108 & $\mathrm{NaN}$ & 4.03000 \\
10 & 0.33319435 & 1.32685 & 0.85500 \\
20 & 0.15333206 & 1.11970 & 5.34000 \\
40 & 0.07575471 & 1.01725 & 5.36000 \\
80 & 0.03762644 & 1.00959 & 2.22500
\end{tabular}

\begin{tabular}{r|r|r|c}
$N$ & $\begin{array}{r}\text { Demyanov } \\
\text { distance }\end{array}$ & $\begin{array}{r}\text { Order } \\
\text { trajectory }\end{array}$ & angle \\
\hline 5 & 1.03202096 & NaN & 0.73000 \\
10 & 0.36562913 & 1.49702 & 3.85000 \\
20 & 0.16060144 & 1.18690 & 3.76000 \\
40 & 0.07933801 & 1.01740 & 4.72000 \\
80 & 0.03952243 & 1.00534 & 4.72000
\end{tabular}

Table VI: Order of Convergence for the modified Euler's method with free selection.
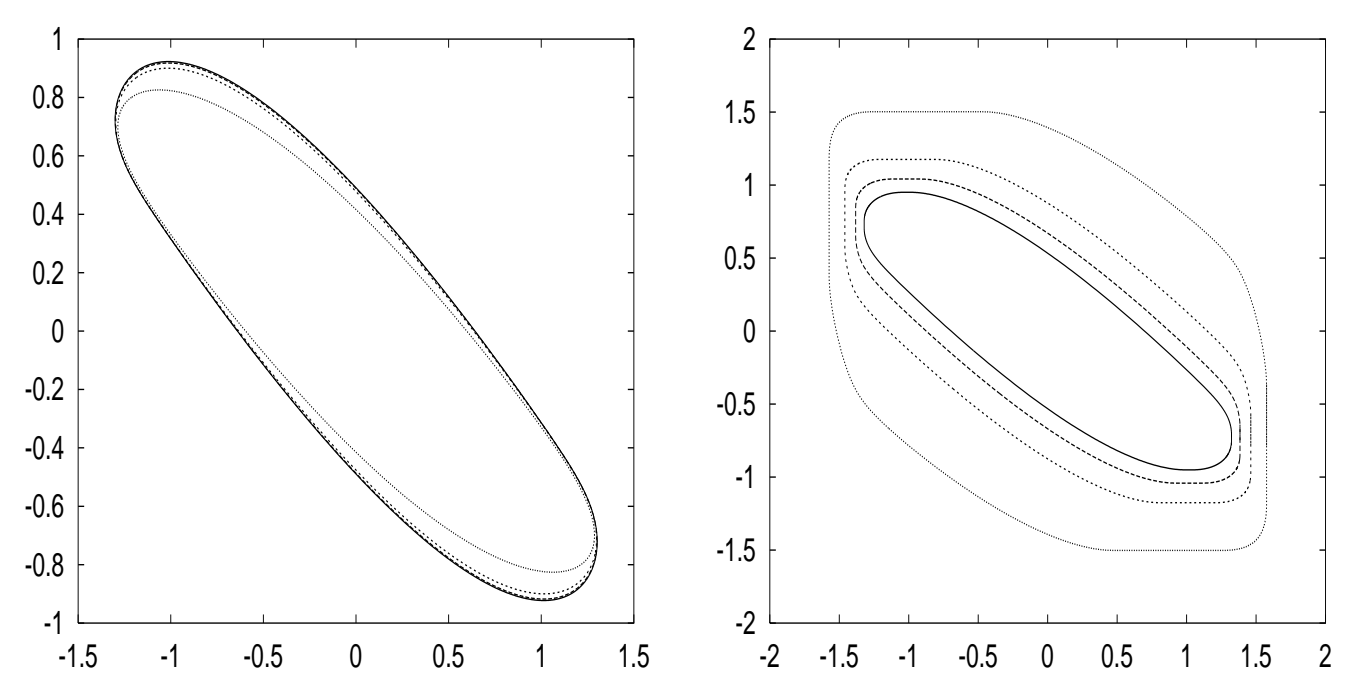

Figure 4: approximations to the reachable set for $N=160$ (solid) and $N=$ 5, 10, 20 (dashed) computed by modified Euler's method with piecewise constant (left) resp. independent selection strategy (iii) (right). 


\section{Outline of Further Research}

It is known that set valued quadrature methods in [4] could lead to a order of convergence greater than two, if the problem satisfies additional smoothness conditions, cf. [3]. In this case, selection strategies with piecewise constant controls are no longer appropriate. Preliminary computer experiments with the classical Runge-Kutta method show that order of convergence greater than two is attainable. But for these Runge-Kutta methods suitable selection strategies have to be studied in more detail. In this context, additional difficulties arise if state constraints are present, because these constraints should be fulfilled also at the intermediate stages of the Runge-Kutta scheme (as in [8]).

Further research can be conducted towards the study of Runge-Kutta schemes as in [29], [13], [27], where the selection strategy is motivated by multiple control integrals. In the special case of two selections per Runge-Kutta step this leads to alternative selection sets of type $\left(u_{a p p}^{(i)}\left(t_{i}+\gamma_{1} h ; \hat{\mathbf{u}}\right), u_{a p p}^{(i)}\left(t_{i}+\gamma_{2} h ; \hat{\mathbf{u}}\right)\right) \in \hat{U} \subset U \times$ $U$, where $U \times U$ corresponds to case (iii) of independent selections in Section 3.2. This set $\hat{U}$ can be described by finitely many nonlinear inequalities and equalities, which can be easily imposed as additional constraints in the discretized optimal control problems.

The proposed method itself can be easily adapted to the calculation of convex reachable sets for nonlinear differential inclusions. For the numerical solution of discretized optimal control problems efficient algorithms are available, cf., e.g., $[6],[16,17]$. In the more general case of nonconvex reachable sets suitable modifications of our approach have to be studied. Theoretical results in this direction can be found in [12], [41], [40] for Runge-Kutta methods of order one and two. A survey of other methods is given in [11] and [8].

However, those Runge-Kutta methods with appropriate selection strategies, which show higher order of convergence in the linear case, are worth being investigated also in the nonlinear case. In addition, these methods have to be compared with set-valued Runge-Kutta methods based on set arithmetics, cf. [8], which work also on the general nonlinear case. First steps in this direction can be found in [8, Example 5.3.1].

\section{References}

[1] J.-P. Aubin and A. Cellina. Differential Inclusions, volume 264 of Grundlehren der mathematischen Wissenschaften. Springer Verlag, BerlinHeidelberg-New York-Tokyo, 1984.

[2] R. J. Aumann. Integrals of Set-Valued Functions. J. Math. Anal. Appl., 12(1):1-12, 1965. 
[3] R. Baier. Mengenwertige Integration und die diskrete Approximation erreichbarer Mengen. Bayreuth. Math. Schr., 50:xxii + 248 S., 1995.

[4] R. Baier and F. Lempio. Computing Aumann's integral. In A. B. Kurzhanski and V. M. Veliov, editors, Modeling Techniques for Uncertain Systems, Proceedings of a Conferences held in Sopron, Hungary, July 6-10, 1992, volume 18 of Progress in Systems and Control Theory, pages 71-92, Basel, 1994. Birkhäuser.

[5] V. I. Blagodatskikh and A. F. Filippov. Differential inclusions and optimal control. In Proc. Steklov Inst. Math., 4, pages 199-259. North-Holland, Amsterdam, 1986.

[6] C. Büskens. Optimierungsmethoden und Sensitivitätsanalyse für optimale Steuerprozesse mit Steuer- und Zustandsbeschränkungen. PhD thesis, Fachbereich Mathematik, Westfälische Wilhems-Universität Münster, 1998.

[7] J. C. Butcher. The Numerical Analysis of Ordinary Differential EquationsRunge-Kutta and General Linear Methods. John Wiley and Sons, Chichester-New York-Brisbane-Toronto-Singapore, 1987.

[8] I. A. Chahma. Set-valued discrete approximation of state-constrained differential inclusions. Bayreuth. Math. Schr., 67:3-162, 2003.

[9] P. Diamond, P. Kloeden, A. Rubinov, and A. Vladimirov. Comparative Properties of Three Metrics in the Space of Compact Convex Sets. SetValued Anal., 5(3):267-289, 1997.

[10] A. L. Dontchev, W. W. Hager, and V. M. Veliov. Second-Order Runge-Kutta Approximations in Control Constrained Optimal Control. SIAM Journal on Numerical Analysis, 38(1):202-226, 2000.

[11] A. L. Dontchev and F. Lempio. Difference methods for differential inclusions: A survey. SIAM Rev., 34(2):263-294, 1992.

[12] A. L. Dontchev and E. M. Farkhi. Error Estimates for Discretized Differential Inclusions. Computing, 41:349-358, 1989.

[13] R. Ferretti. High-Order Approximations of Linear Control Systems via Runge-Kutta Schemes. Computing, 58(4):351-364, 1997.

[14] A. F. Filippov. Differential Equations with Discontinuous Righthand Sides. Mathematics and Its Applications (Soviet Series). Kluwer Academic Publishers, Dordrecht-Boston-London, 1988.

[15] J. E. Gayek. Approximating reachable sets for a class of linear control systems. Internat. J. Control, 43(2):441-453, 1986. 
[16] M. Gerdts. Numerische Methoden optimaler Steuerprozesse mit differentialalgebraischen Gleichungssystemen höheren Indexes und ihre Anwendungen in der Kraftfahrzeugsimulation und Mechanik. volume 61 of Bayreuther Mathematische Schriften, Bayreuth, 2001.

[17] M. Gerdts. Direct Shooting Method for the Numerical Solution of Higher Index DAE Optimal Control Problems. Journal of Optimization Theory and Applications, 117(2):267-294, 2003.

[18] P. E. Gill, W. Murray, M. A. Saunders, and M. H. Wright. User's guide for NPSOL 5.0: A FORTRAN package for nonlinear programming. Technical Report NA 98-2, Department of Mathematics, University of California, San Diego, California, 1998.

[19] J.-B. Hiriart-Urruty and C. Lemaréchal. Convex Analysis and Minimization Algorithms I. Fundamentals, volume 305 of Grundlehren der mathematischen Wissenschaften. Springer, Berlin-Heidelberg-New York-LondonParis-Tokyo-Hong Kong-Barcelona-Budapest, 1993.

[20] J. E. Kelley. The cutting-plane method for solving convex programs. J. Soc. Ind. Appl. Math., 8:703-712, 1960.

[21] K. C. Kiwiel. Methods of Descent for Nondifferentiable Optimization, volume 1133 of Lecture Notes in Math. Springer, Berlin-Heidelberg-New York.Tokyo, 1985.

[22] K. C. Kiwiel. A Constraint Linearization Method for Nondifferentiable Convex Minimization. Numer. Math., 51:395-414, 1987.

[23] A. B. Kurzhanski and I. Vályi. Ellipsoidal Calculus for Estimation and Control. Systems \& Control: Foundations \& Applications. Birkhäuser, BostonBasel-Berline, 1997.

[24] A. B. Kurzhanski and P. Varaiya. Ellipsoidal techniques for reachability analysis: internal approximation. Systems Control Lett., 41:201-211, 2000.

[25] A. B. Kurzhanski and P. Varaiya. On Ellipsoidal Techniques for Reachability Analysis. part I: External Approximations. Optim. Methods Softw., 17(2):177-206, 2002.

[26] A. B. Kurzhanski and P. Varaiya. On Ellipsoidal Techniques for Reachability Analysis. part II: Internal Approximations Box-valued Constraints. Optim. Methods Softw., 17(2):207-237, 2002.

[27] P. E. Kloeden L. Grüne. Higher order numerical schemes for affinely controlled nonlinear systems. Numer. Math., 89:669-690, 2001. 
[28] C. Lemarechal, J. J. Strodiot, and A. Bihain. On a Bundle Algorithm for Nonsmooth Optimization. In O. L. Mangasarian, R. R. Meyer, and S. M. Robinson, editors, Nonlinear Programming 4, pages 245-282, New York, 1981. Academic Press.

[29] F. Lempio and V. Veliov. Discrete Approximations of Differential Inclusions. Bayreuth. Math. Schr., 54:149-232, 1998.

[30] K. Malanowski, C. Büskens, and H. Maurer. Necessary Conditions for Optimal Control Problems Involving Nonlinear Differential Algebraic Equations. In Anthony Fiacco, editor, Mathematical programming with data perturbations, volume 195, pages 253-284. Dekker. Lect. Notes Pure Appl. Math., 1997.

[31] R. Mifflin. A modification and an extension of Lemarechal's algorithm for nonsmooth minimization. Math. Program. Study, 17:77-90, 1982.

[32] R. T. Rockafellar. Convex Analysis, volume 28 of Princeton Mathematical Series. Princeton University Press, Princeton, New Yersey, $2^{\text {nd }}$ edition, 1972.

[33] P. Saint-Pierre. Approximation of the viability kernel. Appl. Math. Optim., 29:187-209, 1994.

[34] K. Schittkowski. On the Convergence of a Sequential Quadratic Programming Method with an Augmented Lagrangian Line Search Function. Math. Operationsforsch. Stat., Ser. Optimization, 14(2):197-216, 1983.

[35] K. Schittkowski. NLPQL: A Fortran subroutine for solving constrained nonlinear programming problems. Ann. Oper. Res., 5:484-500, 1985.

[36] H. Schramm. Eine Kombination von Bundle- und Trust-Region-Verfahren zur Lösung nichtdifferenzierbarer Optimierungsprobleme. Bayreuth. Math. Schr., 30, 1989.

[37] L. M. Sonneborn and F. S. van Vleck. The bang-bang principle for linear control problems. SIAM J. Control, Ser. A, 2(2):151-159, 1965.

[38] P. Varaiya. Reach set computation using optimal control. In M. K. Inan and R. P. Kurshan, editors, Verification of digital and hybrid systems. Proceedings of the NATO ASI, Antalya, Turkey, May 26-June 6, 1997, volume 170 of NATO ASI Ser, Ser. F, Comput. Syst. Sci., pages 323-331. Springer, 2000 .

[39] V. M. Veliov. Approximations to differential inclusions by discrete inclusions. Working Paper WP-89-017, IIASA, Laxenburg, Austria, 1989. 
[40] V. M. Veliov. Second order discrete approximations to strongly convex differential inclusions. Systems Control Lett., 13:263-269, 1989.

[41] V. M. Veliov. Second Order Discrete Approximation to Linear Differential Inclusions. SIAM J. Numer. Anal., 29(2):439-451, 1992.

[42] S. E. Wright. Primal-Dual Interior-Point Methods. SIAM, Philadelphia, PA, 1997.

[43] G. Zoutendijk. Methods of Feasible Directions. Elsevier Publishing Company, Amsterdam, Netherlands, 1960. 